\title{
EXPOSIÇÃO OCUPACIONAL A PRODUTOS QUÍMICOS E AUTOAVALIAÇÃO DA SAÚDE BUCAL: RESULTADOS DA PESQUISA NACIONAL DE SAÚDE 2013
}

\author{
OCCUPATIONAL EXPOSURE TO CHEMICAL SUBSTANCES AND SELF-ASSESSMENT OF ORAL \\ HEALTH: RESULTS OFTHE NATIONAL HEALTH SURVEY 2013
}

Nayara Silva Alves', Mery Natali Silva Abreu², Ada Ávila Assunção

\section{Resumo}

A exposição a produtos químicos no ambiente laboral tem sido associada a condições bucais. $\bigcirc$ objetivo deste estudo foi examinar a associação entre manuseio de produtos químicos e autoavaliação da saúde bucal em uma amostra de trabalhadores brasileiros. Estudo transversal utilizou dados da Pesquisa Nacional de Saúde (PNS), realizada em 2013, no Brasil. A amostra de 36.442 trabalhadores de ambos os sexos e idade $\geq 18$ anos. Foram descritas características socioeconômicas, demográficas, ocupacionais, hábitos de vida e situação de saúde. Para a percepção da saúde bucal foi considerada a variável de desfecho. Para a exposição ocupacional a produtos químicos, a variável explicativa. Análises bivariadas e multivariadas foram desenvolvidas por meio do programa estatístico Stata versão 12.0. Idade, raça/cor e região de residência estavam significativamente associadas à maior prevalência de autoavaliação negativa da saúde bucal. Maior prevalência de autoavaliação negativa da saúde bucal entre os indivíduos expostos a produtos químicos $(R P=|| ,5[1,|0-1,2|]$ para sexo masculino e $R P=\mid, 23[1,|6-|, 3 \mid]$ para o sexo feminino). Essas associações permaneceram significativas no modelo multivariado para ambos os sexos. Concluiu-se que existe maior prevalência de percepção negativa da saúde bucal entre os trabalhadores expostos ao manuseio de produtos químicos no ambiente laboral.

Palavras-chave: Saúde do trabalhador; Exposição ocupacional; Odontologia em saúde pública; Inquéritos de saúde bucal; Estudos transversais.

\begin{abstract}
Exposure to chemicals in the work environment has been associated with oral conditions. The aim of this study was to examine the association between handling chemical products and self-rated oral health in a sample of Brazilian workers. A cross-sectional study used data from the National Health Survey (PNS), carried out in 2013, in Brazil. The sample consisted of 36,442 workers of both sexes and aged $\geq 18$ years. Socioeconomic, demographic, occupational, lifestyle and health status characteristics were described. For the perception of oral health, the outcome variable was considered. For occupational exposure to chemicals, the explanatory variable. Bivariate and multivariate analyzes were developed using the Stata version 12.0 statistical program. Age, race/color and region of residence were significantly associated with a higher prevalence of negative selfrated oral health. Higher prevalence of negative self-assessment of oral health among individuals exposed to chemical products $(P R=1.15[1.10-1.21]$ for males and $P R=1.23[1.16-1.31]$ for the women). These associations remained significant in the multivariate model for both sexes. In conclusion, there is a higher prevalence of negative perception of oral health among workers exposed to handling chemical products in the workplace.
\end{abstract}

Keywords: Occupational health; Occupational exposure; Public health dentistry; Dental health surveys; Cross-sectional studies.

I. Doutoranda em Saúde Pública. Universidade Federal de Minas Gerais, Brasil. (UFMG)

2. PhD em Saúde Pública. Professora Titular da Faculdade de Medicina. Universidade Federal de Minas Gerais, Brasil. (UFMG)

3. PhD em Saúde Pública. Professora Associada na Escola de Enfermagem. Universidade Federal de Minas Gerais, Brasil. (UFMG)

\section{Como citar este artigo:}

Alves NS, Abreu MNS, Assunção AA. Exposição ocupacional a produtos químicos e autoavaliação da saúde bucal: resultados da Pesquisa Nacional de Saúde 20 I 3. Rev Nav Odontol. 2021; 48(2): .05- 6. 


\section{INTRODUÇÃO}

Saúde bucal é uma dimensão essencial da saúde e do bem-estar do indivíduo, pois implica a integridade das estruturas do complexo craniofacial e da mucosa oral, ausência de lesões e de distúrbios bucais e dentários, bem como estar livre de dor orofacial crônica (I). Os agravos à saúde bucal são altamente prevalentes, de maneira a constituírem um problema de saúde pública. Mundialmente, em 2017, foram estimados 3,5 bilhões de casos, com destaque para doença periodontal, cárie e perda dentária (2). Além do impacto sobre o bem-estar e a qualidade de vida das pessoas $(3,4)$ em decorrência das queixas de dor e desconforto, são comuns perturbações em diferentes níveis da funcionalidade humana como mastigação, comunicação e interação social.

Condições bucais consideradas precárias são evitáveis por meio de maior acesso a atendimento odontológico $(1,4)$. Indicadores clínicos de doenças bucais, incluindo cárie dentária, periodontite e câncer orofacial, bem como autoavaliação da saúde bucal (AASB) foram formulados para avaliar essa dimensão da saúde em populações(4). Quanto ao último, estão reconhecidas as suas vantagens para planejar os serviços de saúde pública (5-7). Dentre elas, destaca-se a mudança na ênfase de aspectos puramente biológicos para aspectos psicológicos e sociais (5). A AASB global é uma medida de item único que faz uma pergunta geral sobre a percepção do indivíduo sobre seu estado geral de saúde ou qualidade de vida naquele período específico. As respostas são categorizadas, variando de excelente a ruim (3). A utilização desse indicador deve levar em consideração fatores biológicos, determinantes sociais individuais e contextuais, uma vez que, frequentemente, a literatura tem demonstrado que há grandes diferenças entre os grupos socioeconômicos em relação à AASB (8). Assim, abordagens das condições bucais, além de programas específicos para a população economicamente ativa, são desejáveis, haja vista as evidências sobre a necessidade de cuidados odontológicos na fase adulta da vida (9).

Os adultos inseridos no mercado podem estar expostos aos efeitos das condições de trabalho, que dizem respeito a uma gama de fatores: modelo de gestão e organização da produção, duração da jornada e horários, exposição a agentes biológicos, mecânicos, físicos e químicos $(9,1$ I , I 2). No conjunto, os efeitos dessas condições têm sido associados à prevalência de adoecimento nos grupos de trabalhadores, incluindo as doenças nas gengivas e nos dentes (9, I|-|3). Estresse ocupacional, por exemplo, foi associado à cárie, perda dentária, sensibilidade aumentada a bebidas e alimentos gelados, dor e disfunções temporomandibulares $(|2| 4$,$) .$

$\mathrm{Na}$ literatura odontológica, estão reconhecidos os efeitos de agentes químicos manuseados nos fazimentos laborais sobre os tecidos bucais, como periodontite $(|3| 5$,$) . Exposição à$ poeira de sílica e a névoas ácidas, por sua vez, são fatores etiológicos para a erosão dentária (16). Quando entrevistados, eles associaram esses problemas à precariedade das condições de trabalho. A alta prevalência de cárie dentária foi observada em amostra de trabalhadores da construção civil ( 17). Alta prevalência de dor de dente produziu absenteísmo numa amostra de servidores municipais ( 18). No setor de processamento de carne, maior prevalência de absenteísmo relacionado a dores orofaciais foi identificada no grupo de trabalhadores com AASB negativa se comparada ao grupo com melhor AASB (I I).

Até onde se sabe, no Brasil, ainda não foi publicado um estudo, de abrangência nacional, que tenha investigado a percepção da situação de saúde bucal associada à exposição a produtos químicos no ambiente laboral. À vista disso, tem-se em mente a necessidade de conhecimento generalizável sobre saúde bucal relacionada a exposições ocupacionais.

O objetivo foi examinar a associação entre exposição a produtos químicos no trabalho e a autoavaliação da situação de saúde bucal em uma amostra nacional de trabalhadores. A hipótese para essa investigação é o aumento da chance de AASB negativa quando os indivíduos trabalham expostos a produtos químicos.

\section{METODOLOGIA}

\section{Desenho do estudo}

Trata-se de um estudo observacional de corte transversal que utilizou dados da Pesquisa 
Nacional de Saúde (PNS), realizada em 20 I 3, no Brasil. A PNS é uma pesquisa de base domiciliar, de âmbito nacional, fruto do convênio do Ministério da Saúde com o Instituto Brasileiro de Geografia e Estatística (IBGE). Todos os dados da PNS, incluindo o planejamento amostral, informações técnicas e a divulgação dos microdados, podem ser acessados no endereço https://www.ibge.gov.br/estatisticas/ sociais/saude.html (19). A PNS foi aprovada pela Comissão Nacional de Ética em Pesquisa, do Conselho Nacional de Saúde, em junho de $2013\left(n^{\circ}\right.$ I08538|2.7.0000.0008) (19).

\section{Critérios de inclusão e exclusão}

Na PNS foram selecionados 64.348 domicílios para participar da pesquisa. Destes, 60.202 indivíduos responderam ao questionário individual. As pessoas com pelos menos 18 anos de idade, incluídas na população empregada, foram consideradas elegíveis, ou seja, os adultos empregados que responderam à pergunta EII "Quantos trabalhos tinha na semana de 21 a 27 de julho de 2013 (semana de referência)?". Respondentes desempregados ou que não estavam procurando por emprego foram considerados inelegíveis. A amostra final foi composta por 36.442 indivíduos empregados, de ambos os sexos, ou seja, na semana de referência, estavam trabaIhando em troca de dinheiro ou outro tipo de remuneração, ou auxiliando outra pessoa em seu domićlio ou sem remuneração direta ou, ainda, estavam temporariamente afastados de um trabalho remunerado.

\section{Construção das variáveis}

As variáveis foram divididas em três blocos. No primeiro, foram incluídas aquelas relacionadas às condições socioeconômicas e demográficas (idade, raça/cor, situação conjugal, região geográfica, área de residência, nível de instrução e renda); o segundo bloco englobou as variáveis relativas aos fatores ocupacionais (ocupação, posição na ocupação e exposições ocupacionais). Por fim, no terceiro bloco, foram incluídas variáveis atinentes à situação de saúde (percepção da situação da saúde e saúde bucal, perda dentária, limitação da capacidade para alimentação, escovação dentária, consulta ao dentista, diabetes, tabagismo e consumo de bebidas alcoólicas).

\section{Variável de desfecho: percepção da situação de saúde bucal}

A variável de desfecho, percepção da situação de saúde bucal, foi elaborada de acordo com a resposta obtida à pergunta do módulo sobre saúde bucal: U005 “Em geral, como o(a) sr(a). avalia sua saúde bucal (dentes e gengivas)?": I. Muito boa; 2. Boa; 3. Regular; 4. Ruim; 5. Muito ruim. As respostas foram analisadas por meio de variável dicotômica I. AASB positiva (muito boa e boa); 2. AASB negativa (regular, ruim e muito ruim).

\section{Variável explicativa principal: exposição ocupacional a produ- tos químicos}

A exposição a produtos químicos no ambiente laboral foi considerada variável explicativa principal, sendo baseada na resposta à pergunta MII. "Pensando em todos os seus trabalhos, o(a) sr(a). está exposto(a) a algum destes fatores que podem afetar a sua saúde? A. Manuseio de substâncias químicas": I. não; 2. sim.

\section{Covariáveis}

As variáveis consideradas passíveis de influenciar a associação entre as exposições ocupacionais e a saúde bucal foram: idade (I 8-34, 35-44, 45-59, $\geq 60$ anos); raça/cor autodeclarada (branca e não branca), situação conjugal (vive com e sem cônjuge ou companheiro(a), região geográfica (Sul, Sudeste, Centro-Oeste, Norte e Nordeste), área de residência (urbana e rural). $O$ nível de instrução foi analisado em duas categorias: I. $\geq$ ensino médio completo: pessoas com ensino médio completo; superior completo ou incompleto e pós-graduação; e 2. < ensino médio completo: pessoas sem instrução; fundamental incompleto, completo ou equivalente; ensino médio incompleto ou equivalente.

A renda familiar per capita foi calculada em quatro etapas. Na primeira etapa, foram 
somados os rendimentos brutos mensais dos moradores do domicílio relativos ao trabatho remunerado, aposentadoria ou pensão, pensão alimentícia, aluguel ou arrendamento, seguro-desemprego, benefício assistencial (LOAS, Bolsa Família ou outros programas do governo), rendimentos de caderneta de poupança ou juros de aplicação financeira ou outros rendimentos. Na segunda etapa, a renda familiar total foi dividida pelo número de moradores do domicílio. Em seguida, na terceira etapa, a renda média dos moradores do domicílio foi dividida pelo valor do salário mínimo no Brasil à época. Na etapa final, os participantes foram classificados como pertencendo a famílias com rendimento familiar per capita maior e menor ou igual a I salário mínimo.

A ocupação do respondente foi examinada. As respostas sobre cargo, função, a profissão ou ofício que a pessoa exercia foram agrupadas em quatro categorias, de acordo com a classificação internacional de ocupações, em inglês, International Standard Classification of Occupations (ISCO): I. Gestores e profissionais; 2 . Técnicos e profissionais associados; 3. Trabalhadores de suporte administrativo, serviço e vendas; 4 . Trabalhadores manuais.

Em relação à posição na ocupação, as respostas foram classificadas em quatro categorias: I. Empregado privado (Empregado do setor privado); 2. Empregado público (Militar do exército, da marinha, da aeronáutica, da polícia militar ou do corpo de bombeiros militar e Empregado do setor público (inclusive empresas de economia mista); 3. Trabalhador doméstico (Trabalhador doméstico) e; 4 . Autônomo (Empregador; Conta própria; Trabalhador não remunerado em ajuda a conta própria ou empregador que era membro do domicílio; Trabalhador não remunerado em ajuda a empregado que era membro do domicílio).

Quanto à saúde bucal, obtiveram-se quatro variáveis de interesse: perda dentária $(<13$ dentes ou $\geq$ I 3 dentes); limitação da capacidade para alimentação: ausente (nenhuma) ou presente (leve/moderada, intensa); escovação dentária (pelo menos 2 vezes por dia ou mais de 2 vezes por dia); consulta ao dentista nos últimos 12 meses ( $\lim$ ou não). Foram incluídas variáveis sobre situação geral de saúde (positiva ou negativa), diabetes (não ou sim), tabagismo (nunca fumou, fumante atual e ex-fumante) e consumo de bebidas alcoólicas (não ou sim).

\section{Análise estatística}

Todas as análises foram realizadas por meio do programa estatístico Stata versão I 2.0 e levaram em consideração as ponderações impostas pelo delineamento amostral do estudo (20). Preliminarmente, foram realizadas análises descritivas dos dados, por meio do cálculo das frequências relativas, a fim de se conhecer a distribuição das variáveis em estudo e caracterizar a amostra e a distribuição das variáveis em relação ao gênero. $\bigcirc$ nível de significância adotado foi de $5 \%$, com poder estatístico de $80 \%$. A análise multivariada foi realizada por blocos (bloco I: condições socioeconômicas e demográficas; bloco 2: fatores ocupacionais e; bloco 3: saúde e comportamentos). Adicionalmente, foram estimados os valores de Razão de prevalência (RP), com intervalo de confiança de 95\% (IC 95\%), nas análises bi e multivariada.

\section{RESULTADOS}

A amostra final foi composta por 36.442 (100\%) indivíduos, 47,1\% homens e 52,9\% mulheres. As tabelas I e 2 apresentam a distribuição da amostra (percentual com peso amostral), de acordo com as variáveis de interesse e estratificadas por gênero. Com exceção para as variáveis raça/cor, limitação para a capacidade de alimentação e diabetes $(p>0,05)$, observaram-se diferenciais de gênero.

Predominaram indivíduos com 18 e 44 anos de idade (58,0\%), cor não branca $(51,2 \%)$, vivendo em união $(61,3 \%)$, na região Sudeste do Brasil (43,8\%), em área urbana (87,5\%), com grau de escolaridade $\geq$ ensino médio completo (52\%) e auferindo renda domiciliar per capita $\leq$ I salário mínimo $(72,7 \%)$ (tabela I).

A prevalência de exposição a produtos químicos foi 16,9\%, sendo mais marcante 
Tabela I - Caracterização da população do estudo $(n=36.442)$ segundo variáveis socioeconômicas, demográficas e fatores ocupacionais por gênero, em 2013, PNS, Brasil.

\begin{tabular}{|c|c|c|c|c|}
\hline \multirow[b]{2}{*}{ Variáveis } & \multicolumn{4}{|c|}{ Percentual* } \\
\hline & $\begin{array}{c}\text { Na amostra } \\
\text { total }\end{array}$ & $\begin{array}{l}\text { Entre os } \\
\text { homens }\end{array}$ & $\begin{array}{c}\text { Entre as } \\
\text { mulheres }\end{array}$ & valor-p \\
\hline \multicolumn{5}{|l|}{ Idade (anos) } \\
\hline $18-34$ & 37,5 & 39,1 & 36,0 & $<0,001$ \\
\hline $35-44$ & 20,5 & 19,7 & 21,2 & \\
\hline $45-59$ & 24,1 & 24,6 & 23,6 & \\
\hline$\geq 60$ & 18,0 & 16,7 & 19,2 & \\
\hline \multicolumn{5}{|l|}{ Raça/Cor } \\
\hline Branca & 48,7 & 47,9 & 49,5 & 0,178 \\
\hline Não branca & 51,2 & 52,2 & 50,5 & \\
\hline \multicolumn{5}{|l|}{ Vive com cônjuge } \\
\hline Sim & 61,3 & 67,5 & 55,8 & $<\mathbf{0 , 0 0 1}$ \\
\hline Não & 38,7 & 32,5 & 44,2 & \\
\hline \multicolumn{5}{|l|}{ Região geográfica } \\
\hline Sul & 14,8 & 14,9 & 14,7 & \\
\hline Sudeste & 43,8 & 43,4 & 44,1 & \\
\hline Centro-Oeste & 7,4 & 7,7 & 7,3 & \\
\hline Norte & 7,4 & 26,5 & 7,2 & \\
\hline Nordeste & 26,6 & 47,1 & 26,7 & \\
\hline \multicolumn{5}{|l|}{ Área de residência } \\
\hline Urbana & 87,5 & 85,1 & 89,7 & $<\mathbf{0 , 0 0 1}$ \\
\hline Rural & 12,5 & 14,9 & 10,3 & \\
\hline \multicolumn{5}{|l|}{ Nível de instrução } \\
\hline$\geq$ Médio completo & 52,0 & 46,5 & 56,9 & $<\mathbf{0 , 0 0 1}$ \\
\hline$<$ Médio completo & 48,0 & 53,5 & 43,1 & \\
\hline \multicolumn{5}{|c|}{ Renda domiciliar per capita em salários mínimos** } \\
\hline$>1$ & 27,3 & 31,2 & 23,8 & $<0,001$ \\
\hline$\leq 1$ & 72,7 & 68,8 & 76,2 & \\
\hline \multicolumn{5}{|l|}{ Fatores ocupacionais } \\
\hline \multicolumn{5}{|l|}{ Manuseio de produtos químicos } \\
\hline Não & 83,1 & 80,6 & 85,3 & $<\mathbf{0 , 0 0 1}$ \\
\hline Sim & 16,9 & 19,4 & 14,7 & \\
\hline \multicolumn{5}{|l|}{ Ocupação } \\
\hline Gestores e profissionais & 16,2 & 13,7 & 18,3 & $<0,001$ \\
\hline Técnicos e profissionais associados & 8,1 & 8,6 & 7,7 & \\
\hline $\begin{array}{l}\text { Suporte administrativo, serviços e } \\
\text { vendas }\end{array}$ & 29,7 & 21,1 & 37,3 & \\
\hline Trabalhadores manuais & 46,1 & 56,6 & 36,8 & \\
\hline \multicolumn{5}{|l|}{ Posição na ocupação } \\
\hline Empregado do setor privado & 44,1 & 51,8 & 37,3 & $<0,001$ \\
\hline Empregado público & 14,6 & 10,6 & 18,2 & \\
\hline Trabalhador doméstico & 8,4 & 1,04 & 14,9 & \\
\hline Autônomo & 33,0 & 36,6 & 29,7 & \\
\hline
\end{tabular}

valor-p: teste $\chi^{2}$ para comparação entre os sexos.

*considerando a ponderação amostral.

**Valor do salário mínimo (SM) em 2013: R\$ 678,00. 
entre os homens (19,4\%). A maioria estava inserida em ocupações predominantemente manuais (46,1\%) e estava inserida no setor privado (44, I\%) (tabela I).

Quanto ao desfecho, a prevalência de autoavaliação negativa da saúde bucal foi 30,9\%, (33,0\% homens versus 29,1\% mulheres). Quanto às variáveis da saúde, a maioria (80,3\%) tinha perdido menos de 13 dentes, negou limitação da capacidade para se alimentar (9l, l\%), escovava os dentes pelo me- nos duas vezes por dia (92,9\%), consultou o dentista há menos de um ano (47,8\%), relatou situação de saúde positiva (69,8\%), não relatou diabetes $(94,4 \%)$, nunca havia fumado $(68,1 \%)$ e não consumia bebidas alcoólicas (55,5\%) (tabela 2). Com exceção da doença diabetes, foram observados diferenciais de gênero, em nível significativo (valor $p<0,05$ ), entre as variáveis analisadas.

As prevalências de AASB negativa desagregadas por gênero e os resultados das associações

Tabela 2 - Caracterização da população do estudo $(n=36.442)$ segundo variáveis relativas à saúde, hábitos, em 2013, PNS, Brasil.

\section{Variáveis}

Percepção da situação de saúde bucal

Positiva

Negativa

Perda dentária

$<13$ dentes

$\geq 13$ dentes

Limitação da capacidade para alimentação

Ausente

Presente

Escovação dentária

\begin{tabular}{lcccc}
\hline$\geq 2$ vezes por dia & 92,9 & 89,6 & 95,8 & $<\mathbf{0 , 0 0 1}$ \\
\hline$<2$ vezes por dia & 7,1 & 10,4 & 4,2 & \\
\hline
\end{tabular}

Consulta ao dentista nos últimos 12 meses

\begin{tabular}{lcccc}
\hline Sim & 47,8 & 42,2 & 52,9 & $<\mathbf{0 , 0 0 1}$ \\
\hline Não & 52,2 & 57,8 & 47,1 & \\
\hline Percepção da situação de saúde geral & & & & \\
\hline Positiva & 69,8 & 73,6 & 66,4 & $<\mathbf{0 , 0 0 1}$ \\
\hline Negativa & 30,2 & 26,4 & 33,6 & \\
\hline Diabetes (n=31.975) & & & & \\
\hline Não & 94,4 & 94,7 & 94,2 & 0,360 \\
\hline Sim & 5,6 & 5,3 & 5,8 & \\
\hline Tabagismo & & & & \\
\hline Nunca fumou & 68,1 & 60,7 & 74,7 & $<\mathbf{0 , 0 0 1}$ \\
\hline Fumante atual & 14,7 & 18,6 & 11,2 & \\
\hline Ex-fumante & 17,2 & 20,7 & 14,1 & \\
\hline Consumo de bebidas alcoólicas & & & & \\
\hline Não & 55,5 & 42,1 & 67,5 & $<\mathbf{0 , 0 0 1}$ \\
\hline Sim & 44,5 & 57,9 & 32,5 & \\
\hline
\end{tabular}

valor-p: teste $\chi^{2}$ para comparação entre os sexos.

*considerando ponderação amostral. 
desse desfecho com cada variável em estudo foram observados utilizando o modelo univariado de regressão de Poisson com variâncias robustas (tabelas 3 e 4). A prevalência de AASB negativa é maior no grupo com mais de 34 anos, de raça/cor não branca, vivendo em união (somente para os homens), residentes nas regiões Norte e Nordeste e área rural, com menor nível de escolaridade e renda domiciliar per capita de até I salário mínimo $(p<0,05)$. Observou-se maior prevalência de AASB negativa no grupo que informou realizar trabalhos manuais e na posição de autônomos $(p<0,05)$.

$\mathrm{Na}$ análise multivariada, após os ajustes realizados pelos blocos das variáveis incluídas no

Tabela 3 - Análise bivariada dos aspectos socioeconômicos, demográficos e fatores ocupacionais, de acordo com a percepção da situação de saúde bucal estratificando por gênero ( $n=36.442)$, em 20I3, PNS, Brasil.

\begin{tabular}{|c|c|c|c|c|}
\hline \multirow[b]{2}{*}{ Variáveis } & \multicolumn{2}{|c|}{ Homens } & \multicolumn{2}{|c|}{ Mulheres } \\
\hline & $\begin{array}{c}\% \mathrm{SB} \\
\text { negativa }\end{array}$ & RP [IC 95\%] & $\begin{array}{c}\% \mathrm{SB} \\
\text { negativa }\end{array}$ & RP [IC 95\%] \\
\hline \multicolumn{5}{|l|}{ Idade (anos) } \\
\hline $18-34$ & 27,0 & 1,00 & 23,8 & 1,00 \\
\hline $35-44$ & 35,2 & $1,21[1,15-1,27]$ & 30,3 & $1,22[1,15-1,30]$ \\
\hline $45-59$ & 37,4 & $1,35[1,29-1,42]$ & 32,7 & $1,33[1,26-1,41]$ \\
\hline$\geq 60$ & 37,7 & $1,39[1,29-1,49]$ & 33,0 & $1,27[1,15-1,41]$ \\
\hline \multicolumn{5}{|l|}{ Raça/Cor } \\
\hline Branca & 28,5 & 1,00 & 22,9 & 1,00 \\
\hline Não branca & 37,0 & $1,30[1,23-1,38]$ & 35,6 & $1,42[1,32-1,52]$ \\
\hline \multicolumn{5}{|l|}{ Vive com cônjuge } \\
\hline Sim & 35,3 & 1,00 & 30,4 & 1,00 \\
\hline Não & 28,2 & $0,90[0,86-0,94]$ & 27,4 & $0,96[0,92-1,01]$ \\
\hline \multicolumn{5}{|l|}{ Região geográfica } \\
\hline Sul & 29,0 & 1,00 & 24,3 & 1,00 \\
\hline Sudeste & 27,5 & $0,97[0,90-1,04]$ & 26,1 & $1,04[0,95-1,14]$ \\
\hline Centro-Oeste & 32,0 & $1,07[0,99-1,17]$ & 26,4 & $1,09[0,99-1,20]$ \\
\hline Norte & 43,7 & $1,35[1,26-1,45]$ & 35,0 & $1,35[1,23-1,47]$ \\
\hline Nordeste & 41,3 & $1,38[1,29-1,48]$ & 35,7 & $1,47[1,35-1,59]$ \\
\hline \multicolumn{5}{|l|}{ Área de residência } \\
\hline Urbana & 30,9 & 1,00 & 27,8 & 1,00 \\
\hline Rural & 44,8 & $1,41[1,35-1,47]$ & 39,6 & $1,35[1,27-1,43]$ \\
\hline \multicolumn{5}{|l|}{ Nível de instrução } \\
\hline$\geq$ Médio completo & 24,9 & 1,00 & 23,7 & 1,00 \\
\hline$<$ Médio completo & 40,0 & $1,67[1,61-1,75]$ & 36,1 & $1,70[1,62-1,78]$ \\
\hline \multicolumn{5}{|c|}{ Renda domiciliar per capita em salários mínimos } \\
\hline$>1$ & 25,5 & 1,00 & 20,6 & 1,00 \\
\hline$\leq 1$ & 36,3 & $1,42[1,36-1,49]$ & 31,7 & $1,71[1,60-1,82]$ \\
\hline \multicolumn{5}{|l|}{ Fatores ocupacionais } \\
\hline \multicolumn{5}{|c|}{ Manuseio de produtos químicos } \\
\hline Não & 31,7 & 1,00 & 28,6 & 1,00 \\
\hline $\operatorname{Sim}$ & 38,4 & $1,15[1,10-1,21]$ & 31,9 & $1,23[1,16-1,31]$ \\
\hline \multicolumn{5}{|l|}{ Ocupação } \\
\hline Gestores e profissionais & 20,8 & 1,00 & 19,9 & 1,00 \\
\hline $\begin{array}{l}\text { Técnicos e } \\
\text { profissionais } \\
\text { associados }\end{array}$ & 23,9 & $0,82[0,76-0,88]$ & 19,3 & $0,93[0,87-1,01]$ \\
\hline $\begin{array}{l}\text { Suporte administrativo, } \\
\text { serviços e vendas }\end{array}$ & 29,1 & $1,41[1,24-1,61]$ & 28,4 & $1,62[1,52-1,73]$ \\
\hline Trabalhadores manuais & 38,7 & $1,28[1,23-1,33]$ & 36,3 & $1,33[1,25-1,41]$ \\
\hline \multicolumn{5}{|l|}{ Posição na ocupação } \\
\hline $\begin{array}{l}\text { Empregado do setor } \\
\text { privado }\end{array}$ & 30,3 & 1,00 & 23,8 & 1,00 \\
\hline Empregado público & 24,7 & $1,28[1,15-1,44]$ & 24,4 & $1,20[1,05-1,36]$ \\
\hline Trabalhador doméstico & 33,9 & $1,53[1,41-1,68]$ & 37,5 & $1,60[1,47-1,75]$ \\
\hline Autônomo & 39,1 & $2,04[1,88-2,21]$ & 34,2 & $2,17[2,00-2,36]$ \\
\hline
\end{tabular}

*Percentual (\%) com peso amostral da SB negativa: percepção da situação de saúde bucal negativa (regular, ruim ou muito ruim). 
Tabela 4 - Análise bivariada dos aspectos socioeconômicos, demográficos e fatores ocupacionais, de acordo com a percepção da situação de saúde bucal estratificando por gênero ( $n=36.442)$, em 20I3, PNS, Brasil.

\begin{tabular}{|c|c|c|c|c|}
\hline \multirow[b]{2}{*}{ Variáveis } & \multicolumn{2}{|c|}{ Homens } & \multicolumn{2}{|c|}{ Mulheres } \\
\hline & $\begin{array}{c}\% \text { SB } \\
\text { negativa }\end{array}$ & RP [IC 95\%] & $\begin{array}{c}\% \text { SB } \\
\text { negativa }\end{array}$ & RP [IC 95\%] \\
\hline \multicolumn{5}{|l|}{ Perda dentária } \\
\hline$<13$ dentes & 31,6 & 1,00 & 26,78 & 1,00 \\
\hline$\geq 13$ dentes & 39,5 & $1,38[1,32-1,45]$ & 37,3 & $1,47[1,39-1,55]$ \\
\hline \multicolumn{5}{|c|}{ Limitação da capacidade para alimentação } \\
\hline Ausente & 28,9 & 1,00 & 25,5 & 1,00 \\
\hline Presente & 78,0 & $2,50[2,42-2,60]$ & 63,3 & $2,64[2,53-2,77]$ \\
\hline \multicolumn{5}{|c|}{ Escovação dentária } \\
\hline$\geq 2$ vezes por dia & 30,8 & 1,00 & 28,5 & 1,00 \\
\hline$<2$ vezes por dia & 51,4 & $1,72[1,64-1,80]$ & 45,1 & $1,71[1,58-1,86]$ \\
\hline \multicolumn{5}{|c|}{ Consulta ao dentista nos últimos 12 meses } \\
\hline $\operatorname{Sim}$ & 25,4 & 1,00 & 24,6 & 1,00 \\
\hline Não & 38,4 & $1,48[1,41-1,54]$ & 34,1 & $1,47[1,40-1,54]$ \\
\hline \multicolumn{5}{|c|}{ Percepção da situação de saúde geral } \\
\hline Positiva & 25,7 & 1,00 & 21,3 & 1,00 \\
\hline Negativa & 53,3 & $2,06[1,98-2,14]$ & 44,4 & $2,19[2,09-2,29]$ \\
\hline \multicolumn{5}{|c|}{ Diabetes $(n=31.975)$} \\
\hline Não & 30,8 & 1,00 & 27,8 & 1,00 \\
\hline Sim & 39,7 & $1,33[1,21-1,45]$ & 36,4 & $1,45[1,32-1,60]$ \\
\hline \multicolumn{5}{|l|}{ Tabagismo } \\
\hline Nunca fumou & 27,5 & 1,00 & 26,6 & 1,00 \\
\hline Fumante atual & 44,4 & $1,62[1,55-1,69]$ & 40,3 & $1,43[1,34-1,53]$ \\
\hline Ex-fumante & 38,6 & $1,44[1,37-1,51]$ & 33,1 & $1,27[1,20-1,36]$ \\
\hline \multicolumn{5}{|c|}{ Consumo de bebidas alcoólicas } \\
\hline Não & 32,8 & 1,00 & 30,4 & 1,00 \\
\hline Sim & 33,1 & $1,05[1,01-1,09]$ & 26,2 & $0,97[0,92-1,01]$ \\
\hline
\end{tabular}

*Percentual (\%) com peso amostral da SB negativa: percepção da situação de saúde bucal negativa (regular, ruim ou muito ruim).

Tabela 5 - Análise multivariada avaliando as condições socioeconômicas, demográficas, fatores ocupacionais, situação de saúde e hábitos de vida associados à percepção do estado de saúde bucal, segundo dados da PNS, Brasil, 2013.

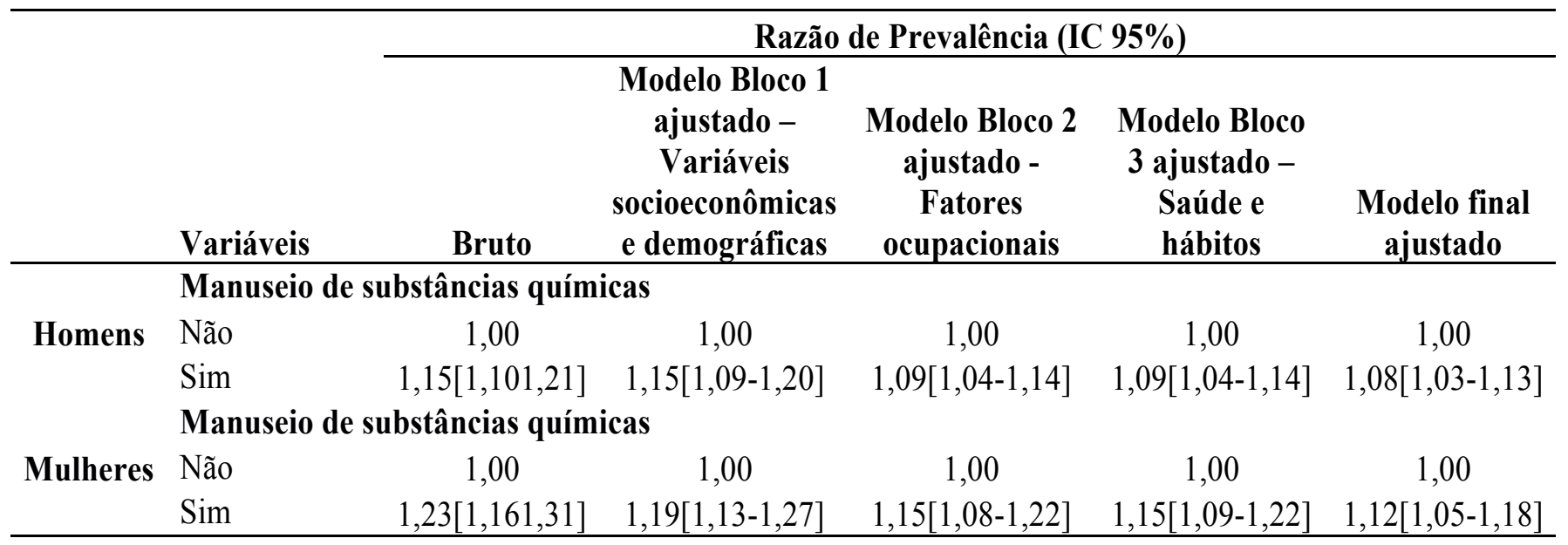

IC 95\% = Intervalo de Confiança de 95\%;Valor-p ajuste do modelo final = I,000.

Bloco I (condições socioeconômicas e demográficas): idade, raça/cor, região geográfica, área de residência, nível de instrução, renda domiciliar per capita em salários mínimos.

Bloco 2 (fatores ocupacionais): ocupação, posição na ocupação.

Bloco 3 (situação de saúde e hábitos de vida): perda dentária, limitação da capacidade para alimentação, escovação dentária, tempo da última consulta ao dentista nos últimos 12 meses, percepção do estado de saúde geral, tabagismo, consumo de bebidas alcoólicas. 
estudo, as covariáveis que permaneceram no modelo final para os homens foram idade, raça/ cor, região de residência, nível de instrução, renda, ocupação, perda dentária, dificuldade para alimentar, frequência de escovação, tempo da última consulta, situação de saúde geral, tabagismo e consumo de álcool. Já para as mulheres foram idade, raça/cor, região de residência, nível de instrução, renda, ocupação, posição na ocupação, dificuldade para alimentar, frequência da escovação, tempo da última consulta, saúde geral e tabagismo. A associação com exposição ocupacional a produtos químicos perdeu a força da associação, mas, permaneceu significativa para ambos os sexos: $R P=1,08[1,03-1,13]$ para os homens e $R P=1,12[1,05-1,18]$ para as muIheres (tabela 5).

\section{DISCUSSÃO}

Em caráter inédito, este estudo representativo da população brasileira evidenciou associação significativa entre AASB e exposição a produtos químicos no ambiente laboral. Esses resultados contribuem para a atualização e para o avanço do conhecimento sobre a saúde bucal da população economicamente ativa no país.Sobre a percepção da saúde bucal, trata-se de um método validado, além de reconhecido como um indicador de saúde conflável $(6,7)$. Quanto à exposição ocupacional a produtos químicos, evidências anteriores foram obtidas de amostras ocupacionais circunscritas, impedindo a generalização e a comparação dos resultados $(9,12,13,16,24)$. Além disso, nenhum estudo perguntou sobre a associação com AASB.

Quase um terço dos trabalhadores avaliaram negativamente a sua saúde bucal, sendo essa prevalência maior nos grupos de homens e mulheres expostos a produtos químicos no trabalho. Uma variedade de produtos manuseados nos ambientes laborais parece ser nociva para os tecidos bucais, entre eles destacam-se ácidos, formaldeído, soda cáustica, solventes, pesticidas, hidrocarbonetos aromáticos policíclicos e cloro. Esses produtos estão presentes na indústria de celuloide, setor de limpeza, tinturarias, fábricas de acumuladores elétricos, extração, fabricação e acabamento de metais, produção de fertilizantes e detergentes, indús- tria vidreira, entre outros setores (| 3, I5,2| ).

Anteriormente, observou-se que a exposição a agentes químicos aumentou 88\% a chance de cárie dentária em operários de seis estados brasileiros (22). Na indústria metalúrgica, foi encontrada alta prevalência de doença periodontal crônica no grupo que, além de exposto a névoas de ácido sulfúrico e clorídrico, informou uso infrequente de fio dental (23). Registrou-se alta prevalência de lesões cervicais não cariosas entre operários expostos a névoas ácidas (24). Em mais de dois terços dos trabalhadores em uma indústria vidreira, foi observada erosão dentária (16). A literatura sobre recortes de gênero nos desfechos da saúde bucal não é convergente $(22,26)$. No Japão, homens tiveram $61 \%$ mais chances de relatar situação de saúde bucal negativa em comparação com as mulheres (26). Em uma amostra de trabalhadores da indústria no Brasil, não houve diferença estatística entre homens e mulheres, no que diz respeito à prevalência de cárie (22).

Sobre a perda da significância nos grupos dos indivíduos com idade mais avançada, quando comparados ao grupo mais jovem, pode-se especular algumas hipóteses. Primeiramente, pessoas mais velhas viveram numa época na qual a deterioração da dentição em idades avançadas foi naturalizada. Perda dos dentes, déficits da função mastigatória, desconforto ou aspectos estéticos, entre outros fatores, teriam sido aceitos como efeitos naturais. Envelhecendo, esses indivíduos com baixa expectativa sobre as suas condições bucais tenderiam a melhor autoqualificar a sua saúde (18). Alerta-se, nesse caso, possível subestimação de AASB negativa entre os mais velhos. Em suma, fatores culturais influenciam a percepção da saúde, conforme já aventado, sendo esperados moduladores da AASB relacionados à geração de uma dada época.

Os efeitos do racismo estrutural, como menor escolaridade e renda, além de menor acesso aos serviços odontológicos, explicariam, ao menos parcialmente, a maior prevalência de AASB negativa nos grupos de raça/ cor não branca, independentemente do gênero (27). No Brasil, os dados do estudo nacional de saúde bucal, SB-Brasil-2003, evidenciaram iniquidades raciais para todos os indicadores 
analisados (cárie, perda dentária, dor e necessidade de prótese) (28).

Maior prevalência de AASB negativa nos grupos com menor escolaridade e renda, independentemente do gênero, confirma a literatura especializada $(18,24,26)$. Quanto maior a escolaridade, maior acesso à informação e maior adesão a práticas preventivas, por exemplo, escovação dentária mais frequente. Anteriormente, identificou-se entre os mais escolarizados maior utilização de serviços odontológicos preventivos quando comparados aos menos escolarizados.

Não surpreende AASB negativa associada à região geográfica, independentemente do gênero. Respondentes residentes nas grandes regiões Norte e Nordeste tiveram maior probabilidade de relatar AASB negativa quando comparados aos da grande região Sul. Resultados semelhantes foram encontrados anteriormente (28). Desigualdades sociais e econômicas inter-regionais explicam os resultados. Historicamente, maior acesso aos serviços de saúde bucal está concentrado nos grupos residentes das grandes regiões Sul e Sudeste $(27,28)$. Além disso, a proporção de adultos que não conseguiu acesso aos serviços odontológicos quando deles necessitava foi maior nas capitais das grandes regiões Norte e Nordeste (27).

Sobre as condições orais dos indivíduos inseridos em ocupações predominantemente manuais, resultado similar foi encontrado pelos autores que avaliaram o impacto da situação socioeconômica sobre essa dimensão da saúde (29). Além das exposições aos riscos laborais, em que estão incluídos produtos químicos, esse grupo aufere menor renda, é menos escolarizado e sofre restrições de acesso a serviços de saúde quando comparados aos outros níveis ocupacionais.

Em relação à posição na ocupação, vale lembrar que na classificação do IBGE, a categoria de autônomos engloba trabalhadores por conta própria; não remunerados em ajuda a conta própria ou empregador que era membro do domicílio (19). A ausência de consenso nos termos atinentes às relações de emprego produz barreiras para comparações. Ainda assim, já foram evidenciados piores resultados de saúde bucal em grupos em situação instável de emprego, como é o caso da categoria "autônomos" no Brasil.

Os limites do estudo devem ser destacados. Em primeiro lugar, a pergunta não inclui a natureza da exposição, ou seja, o questionário da PNS não menciona nem o tipo de produto químico nem a dose e duração da exposição. $O$ delineamento transversal, em segundo lugar, desautoriza especulações sobre causalidade. Por último, diferenças metodológicas entre os estudos que abordaram as associações enfocadas são barreiras para comparações.

tratamento das informações coletadas no domicílio contou com a utilização de pesos amostrais para evitar, por exemplo, sobrerrepresentação de homens ou de mulheres ou de indivíduos residentes em uma região geográfica específica. Além dessa vantagem, a análise beneficiou-se de um cálculo amostral probabilístico adequado ao objetivo de generalizar os resultados para a população de adultos brasileiros. Vale ainda mencionar que o tamanho da amostra viabilizou analisar diferentes covariáveis. Essas vantagens são promissoras quando o desfecho diz respeito à saúde bucal, que é reconhecidamente relacionada a comportamentos de saúde, situação de saúde e fatores do contexto, incluindo as condições de trabaIho $(9,|1-| 3, \mid 6)$.

Os resultados apresentados suscitam estudos para avaliar tendências temporais, bem como esclarecer pontos ainda inexplorados, como os diferenciais de gênero nos desfechos da saúde bucal. Além disso, são pistas para orientar as estratégias de intervenção em saúde bucal em consonância com a realidade da população trabalhadora. Dentro dessa perspectiva, encara-se o desafio de cumprir os princípios da equidade e integralidade em saúde bucal no Brasil. Sistemas de vigilância em saúde bucal focalizados em grupos específicos, como trabalhadores da indústria expostos a produtos químicos, seriam úteis para identificar a magnitude dos problemas, formular e acompanhar as medidas de prevenção. Informações desse tipo são subsídios para o planejamento e ajuste das intervenções em saúde pública. Ou seja, está indicado planejar programas de saúde bucal de modo a alcançar as necessidades de grupos populacionais específicos, por exemplo, trabalhadores expostos a produtos químicos. 


\section{CONCLUSÃO}

A autoavaliação da saúde bucal em grupos ocupacionais foi significativamente associada à exposição laboral em homens e mulheres. Confirmando a hipótese do estudo, observouse que indivíduos expostos a produtos químicos no ambiente de trabalho tiveram maior probabilidade de AASB negativa.

Os autores declaram não haver conflito de interesse.

\section{Autora de correspondência:}

Nayara Silva Alves

Universidade Federal de Minas Gerais (UFMG)

Faculdade de Medicina UFMG. Núcleo de estudos saúde e trabalho.

Rua Alfredo Balena, n 190, $7^{\circ}$ andar, sala 733. Santa Efigênia, Belo Horizonte, Minas Gerais, 303 I 0-450, Brasil.

E-mail: dranayaraalves@gmail.com

\section{REFERÊNCIAS BIBLIOGRÁFICAS}

I. Petersen PE. The World Oral Health Report 2003: continuous improvement of oral health in the 2 Ist century - the approach of the WHO Global Oral Health Programme. Community Dent Oral Epidemiol. 2003;31:3-24 (acesso em I5 jan 2020) Disponível em: https://onlinelibrary.wiley.com/ doi/full// 0. I046/j..2003.com I 22.x?sid=nlm\%3Apubmed

2. Bernabe E, Marcenes W, Hernandez CR, Bailey J, Abreu LG, Alipour V, et al. Global, Regional, and National Levels and Trends in Burden of Oral Conditions from 1990 to 2017: A Systematic Analysis for the Global Burden of Disease 2017 Study. J Dent Res. 2020;99(4):362-73 (acesso em I 5 jan 2020) Disponível em: https://www.ncbi.nlm.nih.gov/pmc/articles/ PMC7088322/

3. Baiju R. Oral Health and Quality of Life: Current Concepts. J Clin Diagnostic Res. 2017;1।(6):ZE21-6 (acesso em I5 jan 2020) Disponível em: https://www.ncbi.nlm.nih.gov/pmcl articles/PMC5535498/

4. Peres MA, Macpherson LMD, Weyant RJ, Daly B, Venturelli

R, Mathur MR, et al. Oral diseases: a global public health challenge. Lancet. 2019;394(10194):249-60 (acesso em I5 jan 2020) Disponível em: https://www.thelancet.com/journals/ lancet/article/PIISO | 40-6736( | 9)3 | |46-8/fulltext

5. Tsakos G, Oliver J. Avaliação das necessidades pela abordagem socio-odontológica. In: Pinto VG, editor. Saúde bucal coletiva. 7th ed. Rio de Janeiro: Guanabara Koogan; 2019. p. 472.

6. Blizniuk A, Ueno M, Zaitsu T, Kawaguchi Y. Association between self-reported and clinical oral health status in
Belarusian adults. J Investig Clin Dent. 2017;8(2):el2206 (acesso em 15 jan 2020) Disponível em: https://onlinelibrary. wiley.com/doi/ I 0. I I I I/jicd. 12206

7. Kim SY, Kim JE, Kim HN, Jun EJ, Lee JH, Kim JS, et al. Association of Self-Perceived Oral Health and Function with Clinically Determined Oral Health Status among Adults Aged 35-54 Years: A Cross-Sectional Study. Int J Environ Res Public Health. 20 I8; I5(8): I68I (acesso em I5 jan 2020) Disponível em: https://www.ncbi.nlm.nih.gov/pmc/articles/PMC6121354/

8. Pattussi MP, Peres KG, Boing AF, Peres MA, Costa JSD. Selfrated oral health and associated factors in Brazilian elders. Community Dent Oral Epidemiol. 20 10;38(4):348-59 (acesso em 25 jan 2020) Disponível em: https://onlinelibrary.wiley. com/doi/ I0. I I I I/j. I600-0528.2010.00542.x

9. Macedo CG, Queluz DP.Quality of life and self-perceived oral health among workers from a furniture industry. Braz J Oral Sci. 201 I;I0(4):226-32 (acesso em I 5 jan 2020) Disponível em:http://revodonto.bvsalud.org/scielo.php?script $=$ sci_ arttext\&pid=S | 677-322520 | | 00040000 |

10. Boing AF, Bastos JL, Peres KG, Antunes JLF, Peres MA. Social determinants of health and dental caries in Brazil: a systematic review of the literature between 1999 and 2010. Rev Bras Epidemiol. 2014;17(suppl 2):102-15 (acesso em I 5 jan 2020) Disponível em: https://www.scielo.br/j/rbepid/a/ jYr5RTyTHPdHG8dFWwkkTmL/?lang=en

I I. Nardi A, Michel-Crosato E, Biazevic MGH, Crosato E, Pizzatto E, Queluz DP. Relationship between orofacial pain and absenteeism among workers in Southern Brazil. Braz J Oral Sci. 2009;8(I):50-4 (acesso em 15 jan 2020) Disponível em:https://periodicos.sbu.unicamp.br/ojs/index.php/bjos/ article/view/8642350

12. Cengiz Mi, Zengin B, İçen M, Köktürk F. Prevalence of periodontal disease among mine workers of Zonguldak, Kozlu District, Turkey: a cross-sectional study. BMC Public Health. 2018;18(I):361 (acesso em I5 jan 2020) Disponível em: https://www.ncbi.nlm.nih.gov/pmc/articles/PMC5857090/

13. Chen WL, Chen YY, Wu WT, Lai CH, Sun YS, Wang CC. Examining relationship between occupational acid exposure and oral health in workplace. BMC Public Health. 2020;20(I):137I (acesso em 15 jan 2020) Disponível em:https://www.ncbi.n/m.nih.gov/pmc/articles/PMC7487460/ 14. Sato Y,Tsuboya T, Aida J, Saijo Y,Yoshioka E, Osaka K. Effortreward imbalance at work and tooth loss: a cross-sectional study from the J-SHINE project. Ind Health. 2020;58( I):26-34 (acesso em I 5 jan 2020) Disponível em: https://www.ncbi.nlm. nih.gov/pmc/articles/PMC69977/3/

15. Vianna MIP, Santana VS. Exposição ocupacional a névoas ácidas e alterações bucais: uma revisão. Cad Saude Publica. 200 |; 17(6): I335-44 (acesso em I 5 jan 2020) Disponível em: https://www.scielo.br/j/csp/a/nHFLvtZXBzNPjQPSj3MsBHx/ abstract/?lang=pt 
16. Chaturvedi P. Assessment of Tooth Wear Among Glass Factory Workers: WHO 2013 Oral Health Survey. I Clin Diagnostic Res. 20I5;9(8):ZC63-6 (acesso em 15 jan 2020) Disponível em: https://uww.ncbi.nlm.nih.gov/pmc/articles/ PMC4576644/

17.Tomita NE, Chinellato LEM, Lauris JRP, Kussano CM, Mendes HJ, Cardoso MTV. Oral health of building construction workers: an epidemiological approach. J Appl Oral Sci. 2005; I3 ( I ):24-7 (acesso em 15 jan 2020) Disponível em: https://www.scielo. br/j/jaos/a/w3Bdr4kXSjwBKwDf3KDymXk/?lang=en

18. Miotto MHMB, Almeida CS, Barcellos LA. Impacto das condições bucais na qualidade de vida em servidores públicos municipais. Cien Saude Colet. 20I4;19(9):3931-40 (acesso em I5 jan 2020) Disponível em: https://uww.scielo.br/j/csc/a/8 VGybkZ9SG7wLQ97zMvHPwf/?lang=pt

19. IBGE [Internet]. Pesquisa nacional de saúde - PNS. Instituto Brasileiro de Geografia e Estatística. 2015 (acesso em 20 jan 2020). Disponível em: https://www.ibge.gov.br/estatisticas/ sociais/justica-e-seguranca/9160-pesquisa-nacional-de-saude. $\mathrm{html}$ ?=\&t=o-que-e

20. StataCorp [Internet]. Stata Statistical Software: Release 12. College Station, TX: StataCorp LLC:; 20II (acesso em 20 jan 2020). Disponível em: https://www.stata.com

21. Awan KH, Hegde R, CheeverVJ, Carroll W, Khan S, Patil S, et al. Oral and pharyngeal cancer risk associated with occupational carcinogenic substances: Systematic review. Head Neck. 20 I8:40( I2):2724-32 (acesso em 15 out 2020) Disponível em: https://onlinelibrary.wiley.com/doi/ I 0. $1002 /$ hed.25486

22. Cangussu MCT, Silva KMG, Mello MV, Vianna MIP, Lima LS. Cárie dentária em trabalhadores da indústria atendidos pelo sesi no brasil e fatores associados. Rev Saúde Coletiva da UEFS. 20 17;6(2):8 (acesso em 15 dez 2020) Disponível em: http:// periodicos.uefs.br/index.php/saudecoletiva/article/view/I I 57 23. Almeida TF, Vianna MIP, Santana VS, Gomes Filho IS. Occupational exposure to acid mists and periodontal attachment loss. Cad Saude Publica. 2008;24(3):495-502 (acesso em 13 mar 2020) Disponível em: https://www.scielo. $\mathrm{br} / \mathrm{j} / \mathrm{csp} / \mathrm{a} / \mathrm{mkSsW} \times \mathrm{Dc} \times 9 \mathrm{GWBy} 5 \mathrm{CP} 6 \mathrm{KMzjc} /$ ?lang=en

24. Bomfim RA, Crosato E, Mazzilli LEN. Relations between oral health and work ability among administrative workers. Braz J Oral Sci. 20I5;|4(I):4I-5 (acesso em I5 jan 2020) Disponível em: https://www.scielo.br/j/bjos/a/3nYXpzSdwyLX P5YDyzhWcrg/?lang=en

25. Assunção AÁ, Abreu MNS, Souza PSN. Exposição a agentes químicos no trabalho no Brasil: resultados da Pesquisa Nacional de Saúde, 20 I3. Rev Saude Publica. 2020;54(92): I - I 3 (acesso em 15 mar 2020) Disponível em: https://www.scielo.br/j/rsp/a/ Zg3fMBNbgcpSCh7QHBMzqY/?lang=pt

26. Tsuboya T, Aida J, Kawachi I, Katase K, Osaka K. Early lifecourse socioeconomic position, adult work-related factors and oral health disparities: cross-sectional analysis of the J-SHINE study. BMJ Open. 20 I 4;4( I 0):e00570 I (acesso em I5 jan 2020) Disponível em: https://www.ncbi.nlm.nih.gov/pmc/ articles/PMC4 I87658/

27. Peres MA, Iser BPM, Boing AF, Yokota RTC, Malta DC, Peres KG. Desigualdades no acesso e na utilização de serviços odontológicos no brasil: Análise do sistema de vigilância de fatores de risco e proteção para doenças crônicas por inquérito telefônico (VIGITEL 2009). Cad Saude Publica. 2012; 28(suppl):90-100 (acesso em 15 jan 2020) Disponível em: https://www.scielo.br/j/csp/a/ wXmvLWT7fTsB8jHMTXYNZjC/?lang=pt

28. Guiotoku SK, Moysés ST, Moysés SJ, França BHS, Bisinelli JC. Iniquidades raciais em saúde bucal no Brasil. Rev Panam Salud Pública. 20 I2;3I(2): I35-4I (acesso em I5 jan 2020) Disponível em: https://www.scielosp.org/article/rpsp/20/2. v3|n2/|35-|4|/pt/

29. Sanders AE, Spencer AJ. Social inequality in perceived oral health among adults in Australia. Aust N Z J Public Health. 2004:28(2):159-66 (acesso em 15 jan 2020) Disponível em:https://onlinelibrary.wiley.com/doi/abs/ | 0.1 I | | /j. I 467842X.2004.tb00930.x?sid=nlm\%3Apubmed 